\title{
Imaging of peripheral nerve tumors
}

\author{
Tarvinder Singh, M.S., AND Michel KLIOT, M.D. \\ Department of Neurological Surgery, University of Washington; and Division of Neurological \\ Surgery, Veterans Administration Puget Health Care System, Seattle, Washington
}

\begin{abstract}
Recent advancements in the field of imaging, particularly magnetic resonance imaging, have improved the way peripheral nerve tumors are diagnosed, monitored, and treated both medically and surgically. When combined with the all-important clinical evaluation and electrodiagnostic studies, imaging permits a clinician to accomplish the following: identify the size, shape, location, and distribution of mass lesions; infer the risks in resecting a mass lesion by displaying its relationship to nearby nerve fascicles; determine growth rates when serial imaging studies are available; suggest the nature of the pathological entity; and visualize muscle denervation associated with nerve damage. The authors describe some of the commonly used imaging modalities and discuss their use in evaluating and treating peripheral nerve tumors and other mass lesions.
\end{abstract}

KEY WORDS • imaging modality - magnetic resonance imaging • peripheral nerve tumor

\section{I} MAGING has played an important role in providing a road map for surgeons in diagnosing, monitoring, and treating tumors. Historically, neuroimaging techniques have been applied to the brain and spine only. Peripheral nerve lesions were diagnosed primarily on the basis of clinical history, physical examination, and electrical studies. Plain film radiography was used to demonstrate secondary skeletal changes in addition to identifying a few types of masses, particularly those with adipose tissue and calcifications, but these techniques provided limited diagnostic information. Advances in CT and ultrasonography have altered the thinking as it became easier to visualize the finer anatomical details of masses, especially when used with the administration of intravenous contrast agents. However, it was not until the introduction of MR imaging, with its increased contrast and resolution for fine depictions of soft tissues and masses, that imaging came to the forefront in the evaluation of peripheral nerve masses. In addition to MR imaging, improved CT and ultrasonography techniques now also provide sufficient resolution and contrast to serve occasionally as less expensive yet valuable tools to complement the study of peripheral nerve lesions.

Despite tremendous advancements in the field of peripheral nerve imaging, limitations remain, especially in the ability to accurately determine the precise lesion type. However, the benefits far outweigh the limitations and the use of imaging to study peripheral nerve tumors has been growing. When used in combination with clinical examination and electrical studies, imaging plays a very important role in the formulation of the treatment plan for the patient suspected of harboring a peripheral nerve mass lesion.

Abbreviations used in this paper: CT = computed tomography; $\mathrm{FOV}=$ field of view; FSE = fast SE; MR = magnetic resonance; $\mathrm{PET}=$ positron emission tomography; $\mathrm{SE}=$ spin echo $; \mathrm{STIR}=$ short tau inversion recovery.

\section{DIAGNOSTIC IMAGING}

Annually an estimated six of every one million people in the United States undergo surgery for peripheral nerve tumors. ${ }^{16}$ More than $90 \%$ of peripheral nerve tumors prove to be benign and originate from one or more of the cell types that comprise peripheral nerves (such as Schwann cells). When these lesions arise from cells within the nerve and cause the nerve to expand gradually, they are called intraneural tumors. Extraneural tumors grow in tissues adjacent to nerves and can eventually compress or infiltrate neighboring peripheral nerves. . $^{3,4,6,7,11,13,20}$ Patients with intraneural and extraneural tumors may present with similar clinical characteristics.

Imaging alone is usually not enough to identify and diagnose peripheral nerve masses accurately. A typical evaluation starts with obtaining a complete clinical history and thorough physical examination. Family history plays an important role in identifying genetic disorders like neurofibromatosis Type 1 and Type 2 . Age, prior medical history of malignancy (such as breast or pulmonary carcinoma), lesions, or radiation therapy can help narrow down the diagnosis to a few tumor types. Physical characteristics such as patches of skin discoloration (café-au-lait spots) or superficial masses (neurofibromas), pigmented spots in the eye (Lisch nodules), and the presence of a Tinel sign over a mass, as well as changes in sensory, motor, and/or autonomic function and reflexes all provide useful clinical information regarding the location and type of tumor.

\section{IMAGING METHODS}

\section{Plain Film Radiography}

Although plain film radiography has limitations in diagnosing peripheral nerve tumors, the initial evaluation of a patient with a suspected soft tissue mass often begins with 

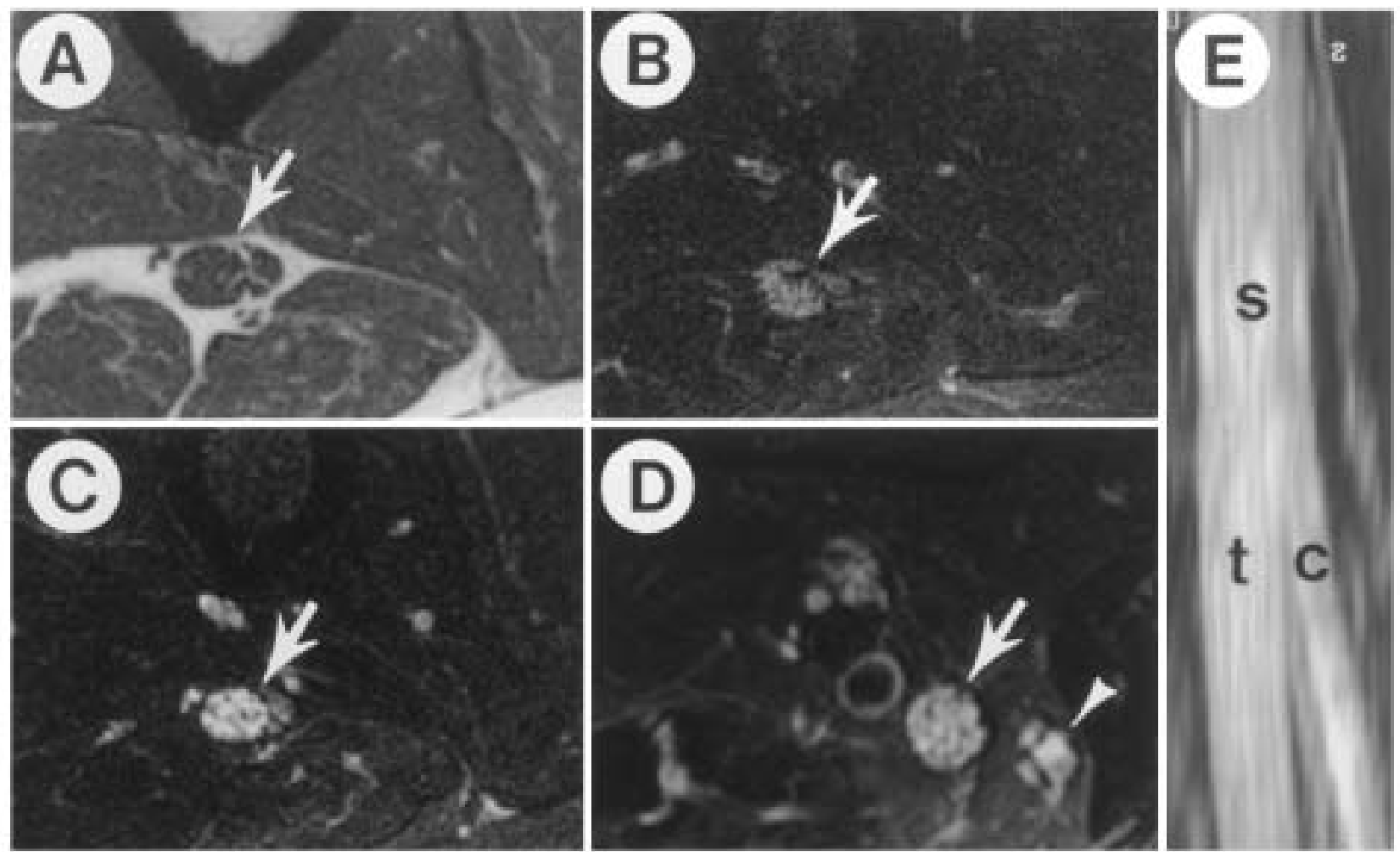

FIG. 1. Magnetic resonance neurography images obtained in a 36-year-old woman with compression neuropathy involving the distal left sciatic nerve near its bifurcation. A: Axial T1-weighted SE image showing the normal fascicular structure of the sciatic nerve (arrow) in the proximal thigh. B: Axial T2-weighted FSE image with the normal signal intensity of fascicles within the sciatic nerve (arrow) in the proximal thigh. C: Axial T2-weighted FSE image showing increased signal and size of fascicles within the sciatic nerve (arrow) in the distal thigh. D: Axial T2-weighted FSE image demonstrating increased signal and size of fascicles within the more distal tibial (arrow) and common peroneal nerve (arrowhead) branches. E: Coronal T2-weighted FSE maximum intensity projection showing the distal sciatic $(s)$ nerve splitting into tibial $(t)$ and common $(c)$ peroneal nerve branches. Reprinted with permission from Kuntz IV et al: Magnetic resonance neurography of peripheral nerve lesions in the lower extremity. Neurosurgery 39(4):750-756, 1996.

this imaging modality. ${ }^{14}$ Radiography is effective in visualizing calcification of some tumor types and/or secondary skeletal changes. For example, a dumbbell-shaped neurofibroma that involves proximal spinal nerves will often deform the adjacent bone structures of the spine, for example, causing an enlarged neural foramen and scalloping of the vertebral body.

\section{Computed Tomography}

Although MR imaging has mostly replaced CT in the evaluation of patients with peripheral nerve tumors, CT is still useful in 1) evaluating patients who are not MR compatible, like those with internal metallic devices such as pacemakers; 2) studying secondary changes in skeletal structures associated with mass lesions, particularly in the spine and chest areas; 3 ) detecting and characterizing calcification in tumors; and 4) evaluating the chest and abdomen for signs of metastasis of peripheral nerve tumors. ${ }^{24}$ When CT scanning is advised, it is usually used with the addition of a contrast agent.

\section{Ultrasonography}

Ultrasonography is among the most widely available and least time-consuming imaging techniques available for the examination of suspected nerve masses. Ultrasonography is safe and causes minimal patient discomfort. The operator can scan long segments of nerves longitudinally, in real time, which would be very time consuming and expensive with MR imaging. Ultrasonography can be particularly useful intraoperatively for localizing tumors that are difficult to see and searching for residual tumors or cystic structures after resection. It is also useful in distinguishing cystic from solid tumors and in visualizing the relationship of major blood vessels to masses. ${ }^{17}$ Similar to MR imaging, ultrasonography may be used to visualize the patterns associated with various tumor types. ${ }^{1,19,23}$ For example, neurofibromas tend to enhance heterogeneously on ultrasonography and have a higher echogenicity in the center than on the periphery. This pattern on imaging, also called the target sign, has been correlated with the benign nature of a tumor. ${ }^{2}$

\section{Positron Emission Tomography}

Positron emission tomography is the tool of choice when it comes to observing metabolic changes. This modality uses radioisotopes, such as fluorodeoxyglucose, to visualize sites of glucose metabolism. Malignant tumors tend to have a higher metabolic rate than benign tumors. 
This property is used in PET scanning to locate potentially malignant masses in peripheral nerves and direct biopsy sampling to the hyperactive areas of a tumor. ${ }^{12}$

\section{Magnetic Resonance Imaging}

Magnetic resonance imaging is usually the modality of choice in preoperative evaluation of peripheral nerve tumors and other types of masses. It provides exceptional contrast of soft-tissue structures, high spatial resolution, multiplanar imaging capability, and no known risks or side effects to patients. When used together with a clinical examination, MR imaging can confirm the presence of a mass and visualize the fine anatomical details of involved peripheral nerves. Magnetic resonance neurography ${ }^{8,9,15}$ comprises a series of techniques that further enhance the contrast between peripheral nerves and surrounding tissues. By employing special pulse sequences, high-field strength scanners, and multiple phased-array coils optimized for a particular body region, an improved signal-tonoise ratio can be achieved, leading to a higher spatial resolution and greater contrast while maintaining an adequate FOV (Fig. 1). This improved contrast and resolution helps greatly in determining the precise location, size, shape, and resectability of peripheral nerve masses (Figs. 2-6). Additionally, 3D reconstruction algorithms are useful in helping the clinician to visualize the tumor's entire extent and geometry. For example, MR neurography makes it possible to distinguish the more homogeneous appearance of tumors and cysts from the characteristic fascicular structure of normal nerves (Fig. 2).

Peripheral nerve masses are generally visualized in two or more orthogonal planes to obtain a longitudinal and a cross-sectional view of the nerve and/or the tumor. Although an in-plane longitudinal view offers a better perspective on the span of the tumor, the cross-sectional plane displays the size and relationship of the tumor to the fascicles of the affected nerve (Figs. 3 and 4). Combining images from these two planes gives an idea of the tumor shape. The choice of exact image orientation usually depends on the anatomical region under examination. For masses in the extremities and spine, axial T1- and T2-weighted images are common; coronal and oblique sagittal planes are typically used for visualizing brachial plexus structures.

Despite the availability of many different MR imaging pulse sequences, most peripheral nerve tumors are evaluated using a combination of a few T1- and T2-weighted pulse sequences. Because the fat present in the thin layer of perineurium and internal epineurial tissue appears bright, T1-weighted images (short TR and TE) show finer anatomical details, such as the fascicular structures of nerves. The images appear isointense to the neighboring normal muscle tissues. When used in combination with contrast agents like Gd, T1-weighted images can accentuate the boundaries between tumor tissue and nerve fascicles (Fig. 3) and are also useful in distinguishing between solid, cystic, and necrotic areas of tumors.

Pathological changes in nerves and mass lesions are best shown on T2-weighted (long TR and TE) images (Fig. 1). In T2-weighted images, normal peripheral nerves appear isointense to slightly hyperintense relative to normal muscle. Additionally, fat-suppressed FSE or STIR techniques demonstrate increased sensitivity to free water content and

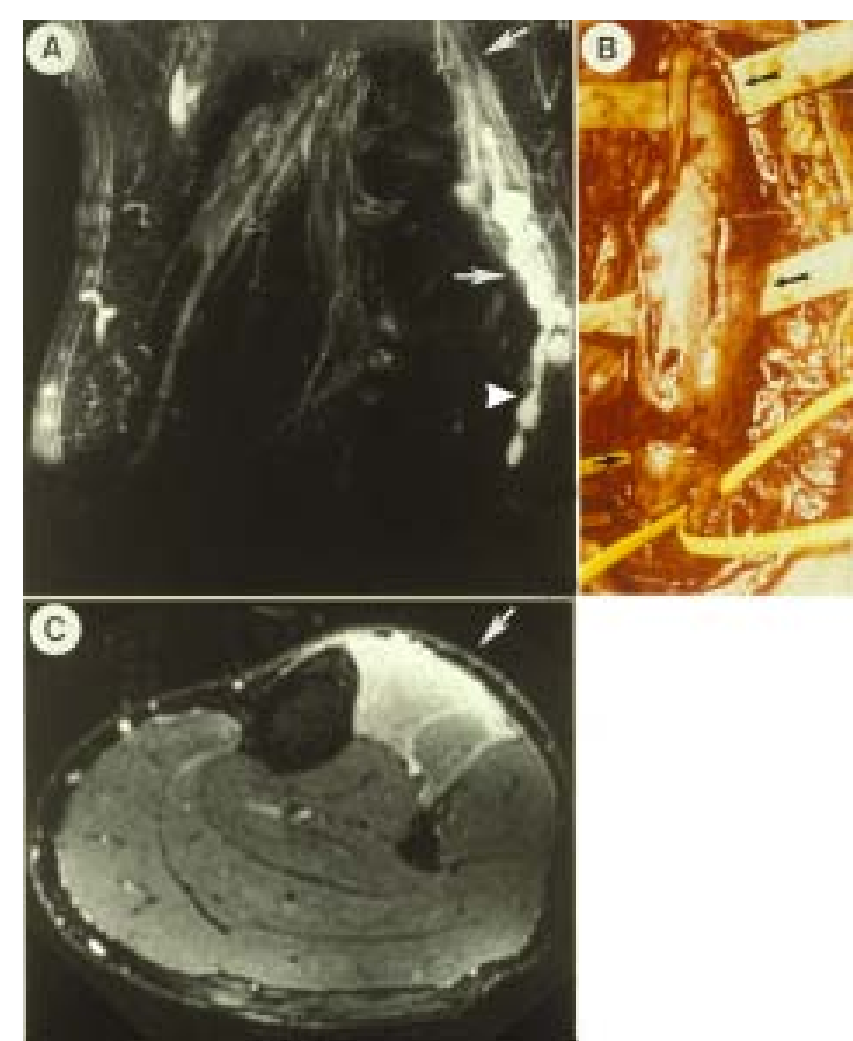

FIG. 2. Images obtained in a 40-year-old man who presented with left peroneal nerve palsy and a lower-leg mass adjacent to the fibular head. A: Coronal T2-weighted MR neurography FSE multiplanar reconstruction showing multiple high signal intraneural cysts within the distal common peroneal nerve (between white arrows) that extends into the deep peroneal nerve branch (arrowhead). Note the abnormally bright nerve fascicles (between white arrows) splayed apart by the cysts. B: Intraoperative photograph demonstrating abnormal enlargement of the common peroneal nerve (middle black arrow) compared with its normal size more proximally (upper black arrow). Note the cyst's extension into the deep peroneal branch (lower black arrow). C: Axial T2-weighted MR neurography image with fat suppression demonstrating increased signal in denervated muscles (white arrow) supplied by deep peroneal nerve branch. Adapted with permission from Kuntz IV et al: Magnetic resonance neurography of peripheral nerve lesions in the lower extremity. Neurosurgery 39(4):750-756, 1996.

are useful in highlighting even subtle tissue abnormalities caused by tumors. By suppressing the hyperintense fat signal, lesion to background contrast is enhanced, making it easier to locate peripheral nerve tumors (Fig. 4).

The exact choice of a specific pulse sequence is based on the balancing of factors such as scan time, spatial resolution, contrast, motion artifacts, and signal-to-noise ratio. Conventionally, standard T1 and T2 FSE images are obtained in all cases and are often enough to evaluate peripheral nerve masses. Other imaging techniques like the STIR sequence and T2 FSE with frequency-selective fat saturation provide suppression of signal from fat tissue, thus increasing the conspicuity of nonfat tissues such as mass lesions. However, they suffer from lower signal-tonoise ratio and uneven fat suppression across the FOV, respectively. ${ }^{22}$

To visualize the relationship between different tissue 

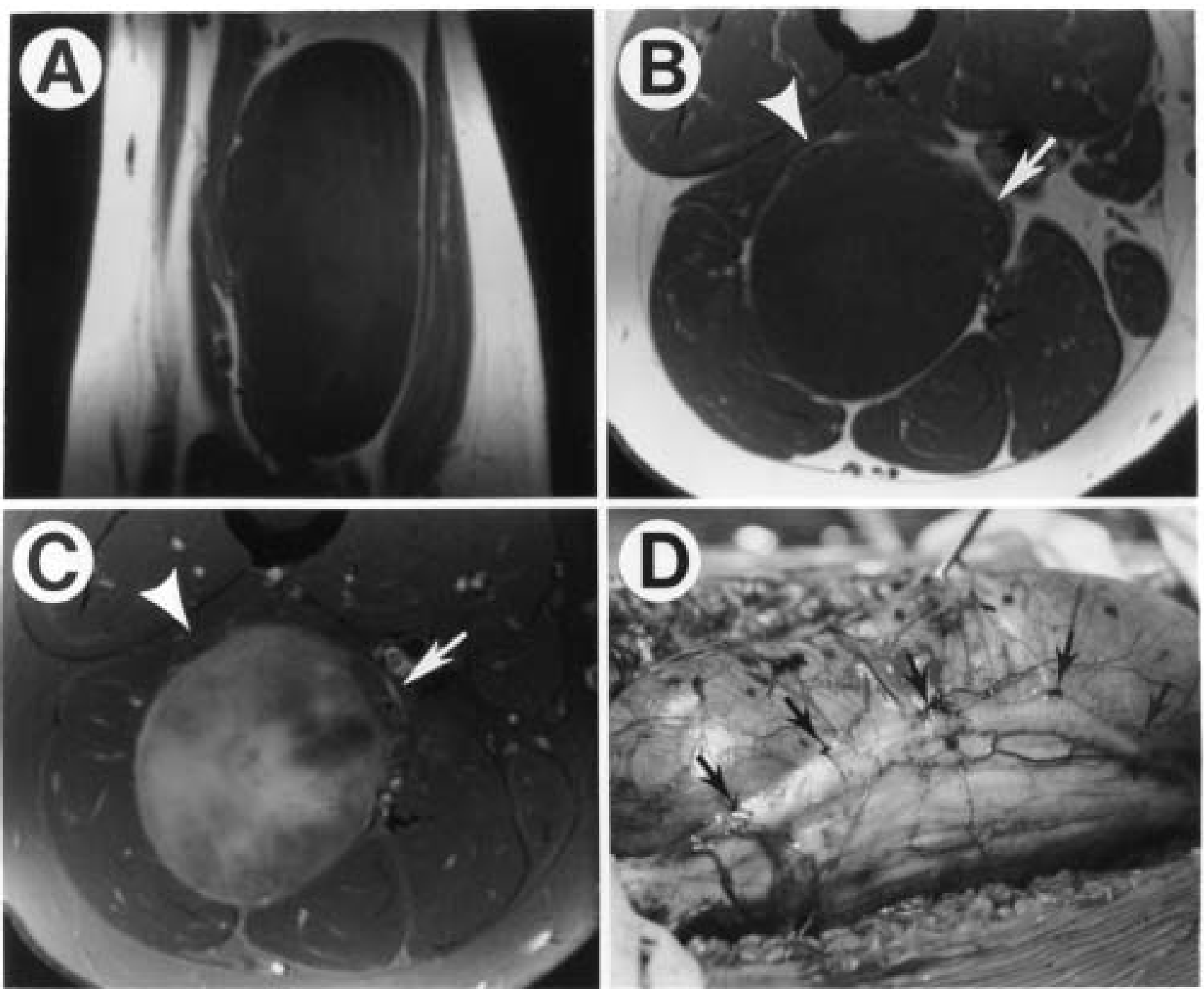

FIG. 3. Images obtained in 20-year-old woman with neurofibromatosis Type 1 who presented with an enlarging symptomatic right sciatic nerve mass, presumably a neurofibroma, above the popliteal fossa. A-C: Magnetic resonance neurography images of a large right sciatic nerve neurofibroma just above the popliteal fossa. A: Coronal T1-weighted SE image showing a large, oval, sciatic nerve neurofibroma with a low signal. B: Axial T1-weighted SE image without contrast enhancement. C: Axial T1-weighted SE image after the administration of Gd showing the fascicular structure of the common peroneal nerve (white arrowhead) and the tibial nerve (white arrow) split apart along the anterior and lateral circumference of the low signal sciatic nerve tumor. D: Intraoperative photograph demonstrating the posterior lateral aspect of the tumor. The black arrows overlie the tumor and point to small sutures placed to demarcate the border between tumor (above) and functioning common peroneal nerve fibers (below) identified with intraoperative electrophysiological stimulation.Reprinted with permission from Kuntz IV et al: Magnetic resonance neurography of peripheral nerve lesions in the lower extremity. Neurosurgery 39(4):750-756, 1996.

types, T1- and T2-weighted images are obtained exactly at the same location and plane using the same FOV. This allows the radiologist to match the fine anatomical nerve features seen on T1-weighted images with the pathological tissue features seen on T2-weighted images. ${ }^{22}$

Most peripheral nerve sheath tumors appear isointense or slightly hyperintense on T1-weighted images and hyperintense on $\mathrm{T} 2$-weighted images relative to the signal intensity of neighboring normal muscle tissue (Figs. 3-5). Although it is possible to visualize the nerve fascicles in relationship to intra- and extraneural tumors, as well as to create a 3D representation of the tumor, determination of the exact tumor type or its origin is still usually difficult, if not impossible, based solely on imaging data. Certain peripheral nerve masses do present unique characteristics. ${ }^{18,26}$ The majority of tumors show enhancement following administration of contrast agents like Gd (Figs. 3 and 4). Two-thirds of neurofibromas display increased central and decreased peripheral signal intensity on T1weighted images (the "target sign") and an opposite pattern of increased peripheral and decreased central signal intensity on T2-weighted images. ${ }^{21,27}$ Additionally, hyper- 

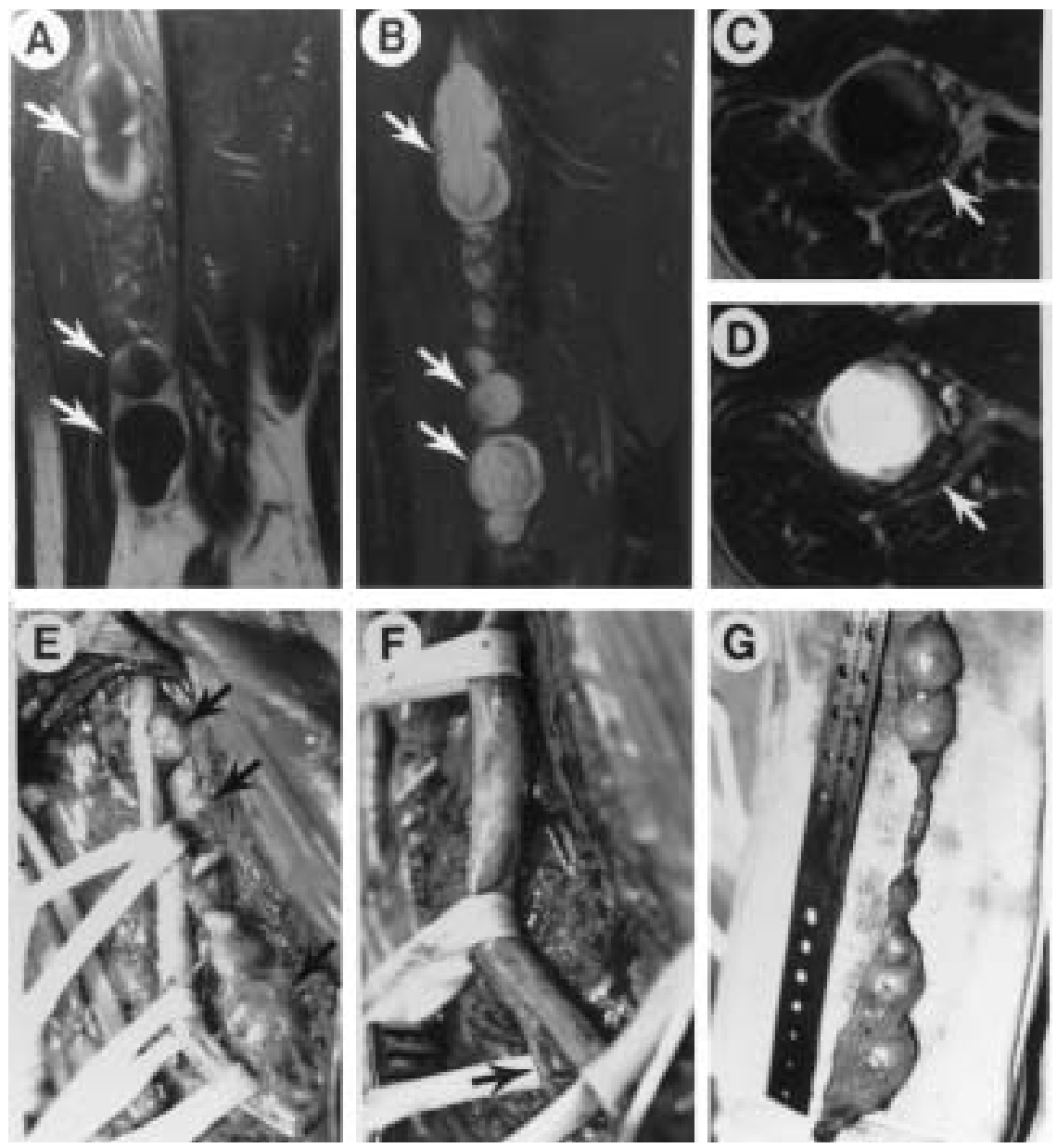

FIG. 4. Images obtained in a 69-year-old woman with a symptomatic mass involving the right sciatic nerve in her lower thigh. Magnetic resonance neurography (A-D) and intraoperative photographs $(\mathrm{E}-\mathrm{G})$ of the right sciatic nerve and mass. A: Coronal fat-suppressed T1-weighted SE image after Gd administration showing heterogeneous enhancement of a multilobulated and cystic mass (white arrows) in the posterior thigh. B: Coronal STIR FSE image showing high signal enhancement within the multilobulated lesion (white arrows). C: Axial T1-weighted SE image after administration of Gd. D: Axial T2-weighted FSE image showing discrete demarcation of the fascicular structure of the sciatic nerve (white arrow) along the posteromedial circumference of the tumor. E: Intraoperative photograph showing Penrose loops elevating the sciatic nerve from the underlying multilobulated neurofibroma (black arrows). F: Intraoperative photograph obtained after removal of the neurofibroma. Note the splitting of the distal sciatic nerve into common peroneal and tibial (black arrow) branches. G: Photograph of resected neurofibroma oriented as shown in A and B.

plastic lymph nodes may resemble tumors and enhance similarly, making it difficult to distinguish them from each other. In contrast, ganglion cysts, ${ }^{28}$ which do not enhance, appear unusually hypointense on T1-weighted images, and hyperintense on T2-weighted images and can thus be distinguished from solid masses (Fig. 2). ${ }^{28}$

Despite many advances in technology and technique, MR imaging is still limited in its ability to identify aspects of tumor pathology such as tumor grade or malignancy accurately. 5,18,26,27 Picking up small changes in size or volume of mass lesions is also difficult on MR imaging. In addition, the appearance and intensity of images obtained with MR imaging may change from one patient to another and between multiple runs on the same patient. Magnetic resonance imaging can also suffer from motion artifacts, uneven fat suppression, and poor signal-to-noise ratio. Therefore, evaluation of differences in image intensity can be difficult to interpret. Sometimes it is useful to make semiquantitative determinations by using a ratio of signal intensity in the suspected pathological areas to that of the adjacent normal tissues. Software algorithms can also help in synchronizing the T1- and T2-weighted images and in determining the approximate size of the mass.

\section{CLINICAL APPLICATION}

The evaluation of a peripheral nerve tumor usually entails a multipronged approach, with patient complaints, clinical findings, electrodiagnostic test results, and imaging studies all playing a critical role. Imaging data obtained and analyzed at different points during a patient's clinical course provide important information on the location, size, shape, rate of growth, and resectability of a mass that helps in determining the proper course of treatment. $3,4,10,12,27$

High-resolution MR imaging of nerves (MR neurogra- 

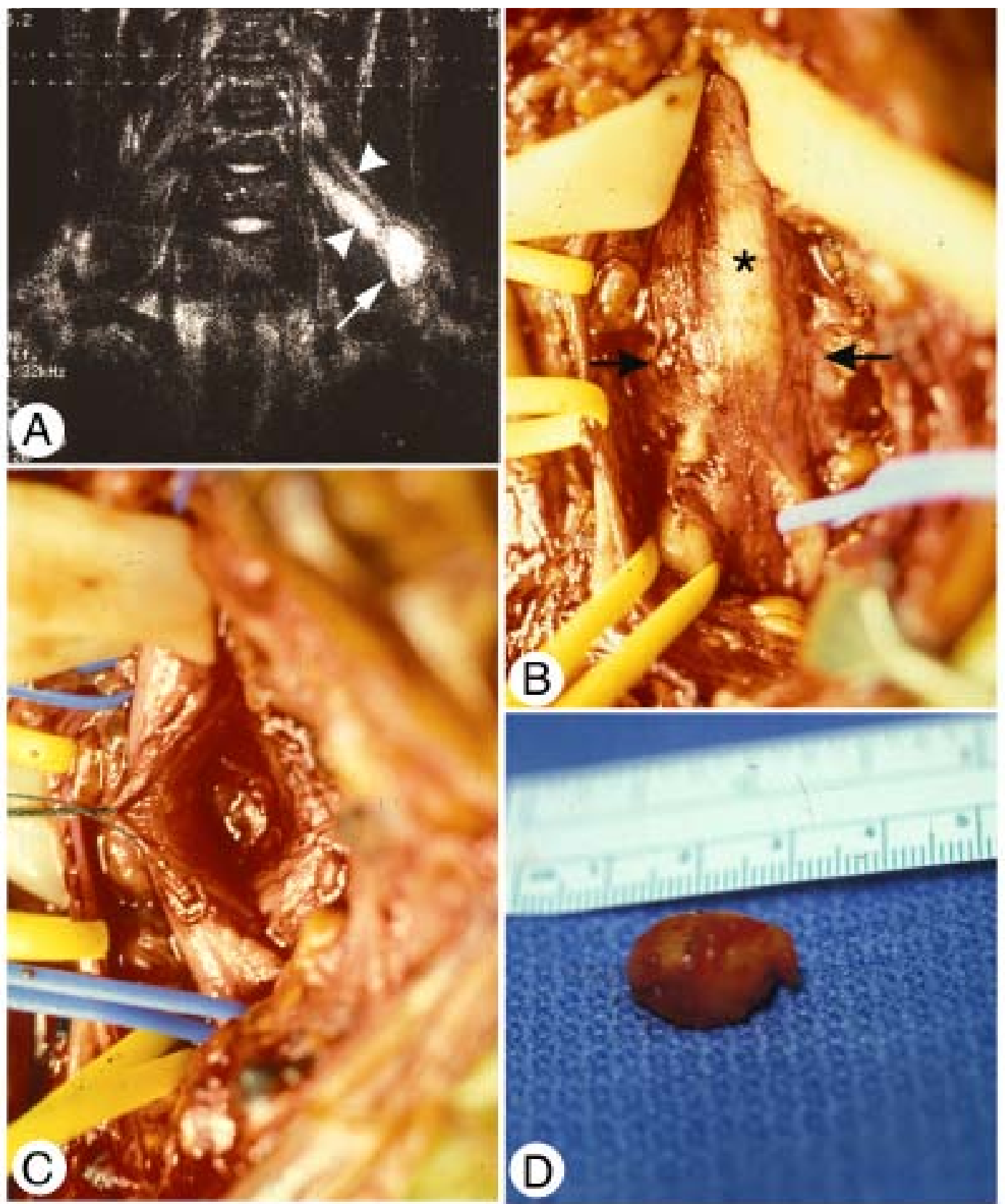

FIG. 5. Images obtained in a 37-year-old woman with a symptomatic mass involving the upper trunk of her left brachial plexus. A: Magnetic resonance neurography and coronal STIR image through the neck of the patient. Note the abnormally high signal intensity involving the C-5 (upper white arrowhead) and dilated C-6 (lower white arrowhead) spinal nerves, which converge to form the upper trunk. Note the distortion of the upper trunk by a bulbous mass with high-signal intensity (white arrow). B: Intraoperative photograph of the upper trunk of the brachial plexus showing the functional nerve fibers of the upper trunk (black asterisk) on the surface of the underlying nerve sheath tumor (between the arrows). C: Intraoperative photograph obtained after resection of the left upper trunk mass leaving a cavity along its lateral side. D: Photograph of the resected well-encapsulated mass taken out in a single piece which was shown to be a schwannoma after biopsy sampling.Reprinted with permission from Kwok et al: Resection of benign brachial plexus nerve sheath tumor using intraoperative electrophysiological monitoring. Neurosurgery Video 60 Suppl 2 (ONS Suppl 2):316-321, 2007.

phy) is used to visualize the relationship of a tumor to the neighboring tissues. Preoperative imaging studies can pinpoint the location and size of the tumor, determine the intraneural or extraneural nature of the tumor, visualize its relationship to peripheral nerve fascicles to assess the chances of resecting the tumor without disturbing function, and determine the best surgical approach (Figs. 3-5).

Magnetic resonance imaging is also useful in delineating the relationship of a peripheral nerve tumor to other adjacent structures such as major blood vessels and inter- 


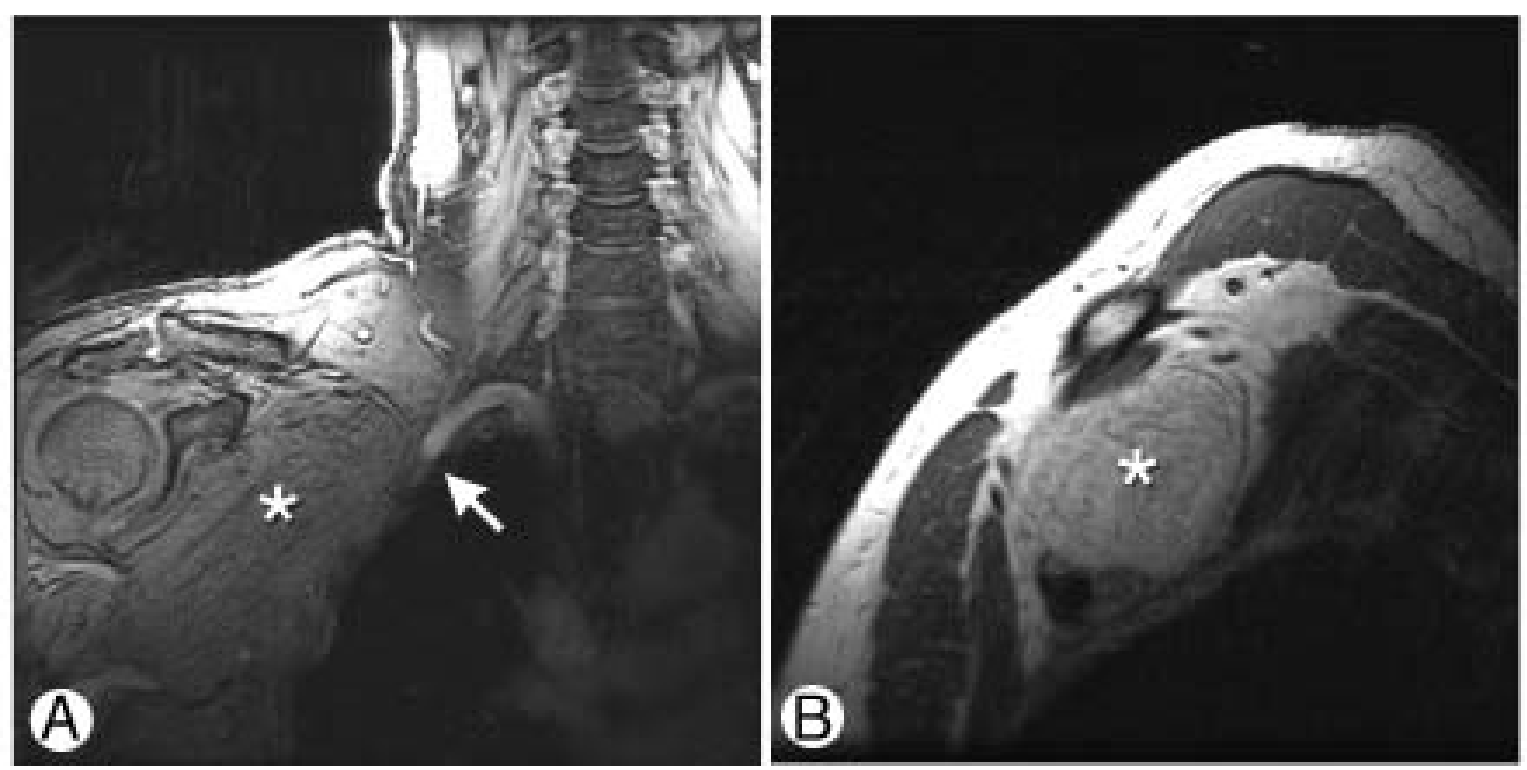

FIG. 6. Magnetic resonance images obtained in a 49-year-old, right-hand-dominant man with a symptomatic and progressively enlarging mass in his right axillary region. Note the very large mass (asterisk) that appears to displace the right brachial plexus and vascular elements inferiorly (white arrow). A: Sagittal T1-weighted image showing that the mass has high signal characteristics of fat tissue (asterisk). B: Coronal fat-suppressed Gd-enhanced T1-weighted gradientecho image demonstrating patchy enhancement of the mass (asterisk). These imaging features are consistent with the diagnosis of lipoma.

nal organs. For example, MR imaging of the pelvis can be used to appreciate whether or not a peripheral nerve mass is impinging on the ureter of the kidney. Displacement or encasement of the ureter and, if present, hydronephrosis can be seen in the images. If involvement of the ureter is suspected, then preoperative stenting can be performed to minimize the risk of surgically lacerating the ureter during tumor dissection and resection. Magnetic resonance imaging can also be used to demonstrate the relationship of an extraneural mass to important adjacent peripheral nerves and vascular structures (Fig. 6).

Malignant peripheral nerve tumors are rare and hard to distinguish from benign nerve masses preoperatively on the basis of clinical symptoms and imaging characteristics. $^{5}$ Definitive diagnosis of a malignancy is only achievable through the pathological analysis of a tumor biopsy specimen. However, certain features suggest malignancy but do not prove it, including evidence of metastasis, rapid growth, size greater than $5 \mathrm{~cm}$, increased metabolic activity on a PET scan, and a history of having malignant peripheral nerve tumor(s) (Fig. 7). In contrast, many benign peripheral nerve tumors either reach a point where they stop growing or grow very slowly. Rarely, such tumors also regress. Serial imaging studies thus provide important information on the pathological grade and growth course of a tumor (Fig. 8).

Magnetic resonance imaging can also help to distinguish between recurrent tumor and radiation-induced plexitis or neuritis. Although recurrent tumors usually present on imaging with hyperintense T2-weighted signal and focal and irregular enlargement of the associated nerve(s), radiation-induced plexitis or neuritis appears as uniform enlargement or narrowing of the involved nerve(s) with a diffuse pattern of increased T2 signal (Fig. 9).

Intraoperative ultrasonography can aid in locating and resecting mass lesions in cases of multinodular or multiple tumors, as well as in distinguishing tumors from functioning and/or normal-appearing nerve fascicles. ${ }^{25}$ Ultrasonography has also been used to guide the percutaneous biopsy of a tumor suspected of being malignant. However,

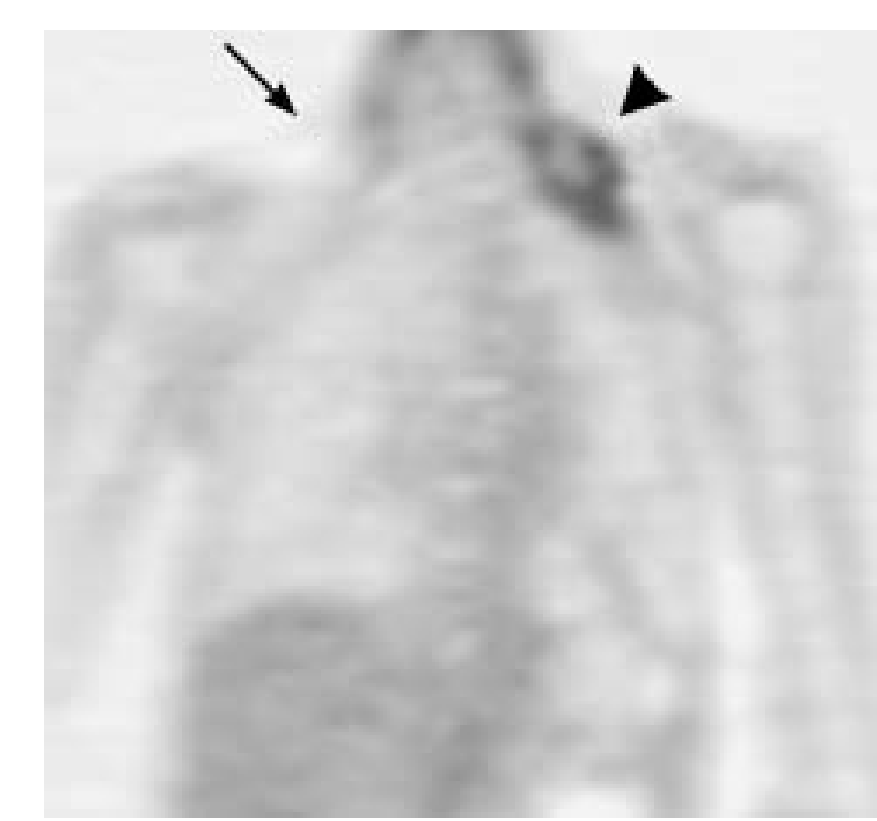

FIG. 7. Positron emission tomography image of a malignant neurofibroma involving the left brachial plexus in a 29 -year-old man with neurofibromatosis Type 1 . Note the increased metabolic rate shown by the darker area (arrowhead) in the left shoulder as compared with the lighter area in the right shoulder (black arrow). Also note the lower metabolic activity in the center of the left brachial plexus mass, probably caused by necrosis or hemorrhage. 

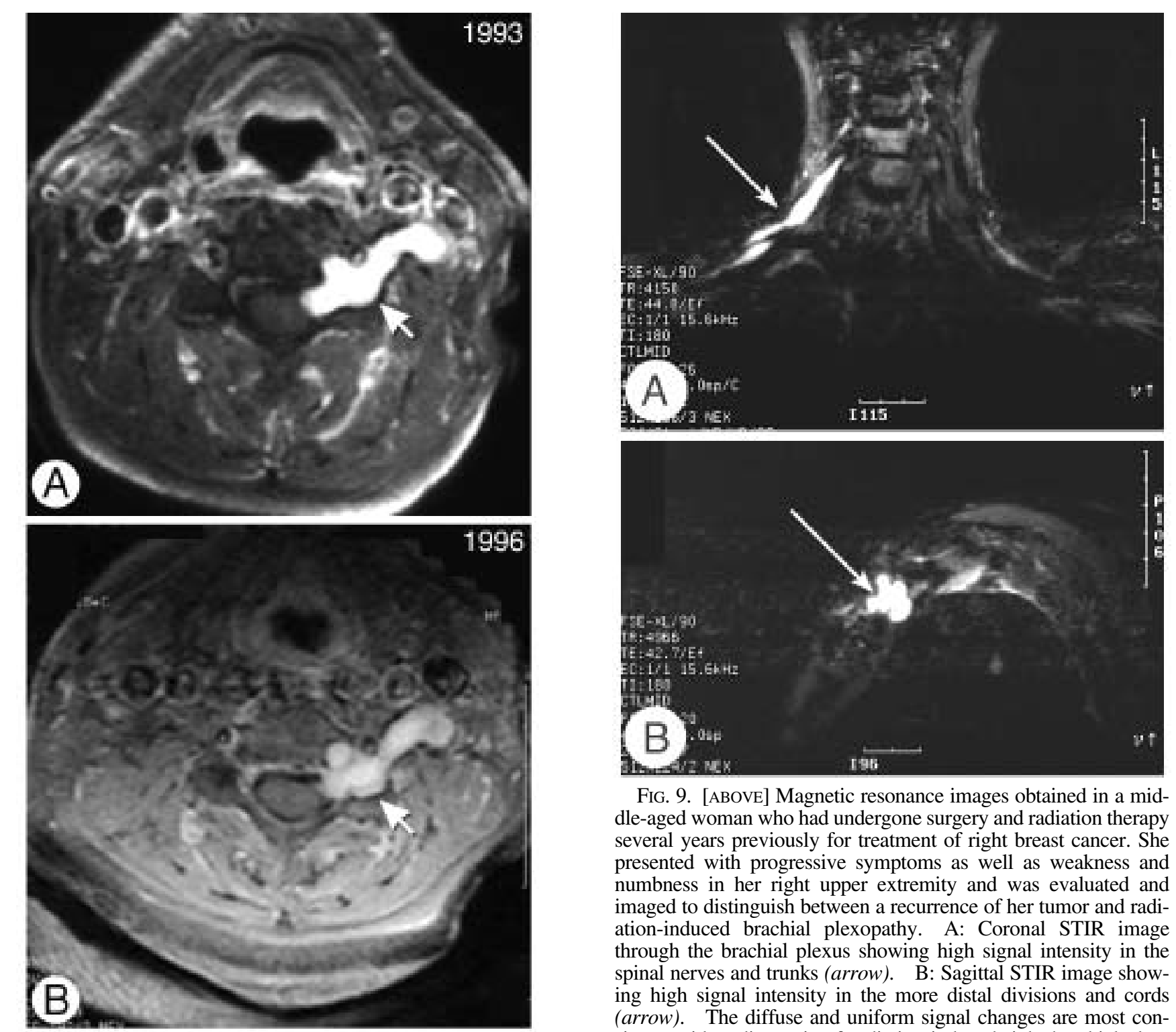

FIG. 9. [ABOVE] Magnetic resonance images obtained in a middle-aged woman who had undergone surgery and radiation therapy several years previously for treatment of right breast cancer. She presented with progressive symptoms as well as weakness and numbness in her right upper extremity and was evaluated and imaged to distinguish between a recurrence of her tumor and radiation-induced brachial plexopathy. A: Coronal STIR image through the brachial plexus showing high signal intensity in the spinal nerves and trunks (arrow). B: Sagittal STIR image showing high signal intensity in the more distal divisions and cords (arrow). The diffuse and uniform signal changes are most consistent with a diagnosis of radiation-induced right brachial plexopathy. No evidence of recurrent tumor is seen. Reprinted with permission from Grant GA et al: The utility of magnetic resonance imaging in evaluating peripheral nerve disorders. Muscle Nerve 25:314-331, 2002.

FIG. 8. [LEFT] Axial fat-suppressed Gd-enhanced T1-weighted MR images obtained in a patient with an unoperated mass involving the left C-5 spinal nerve and having an appearance consistent with a diagnosis of intradural and extradural benign nerve sheath tumor (schwannoma or neurofibroma). Images of mass (arrows) taken on three occasions in 1993 (A), 1996 (B), and 2005 (C). Note the absence of significant growth over the 12-year time period. 

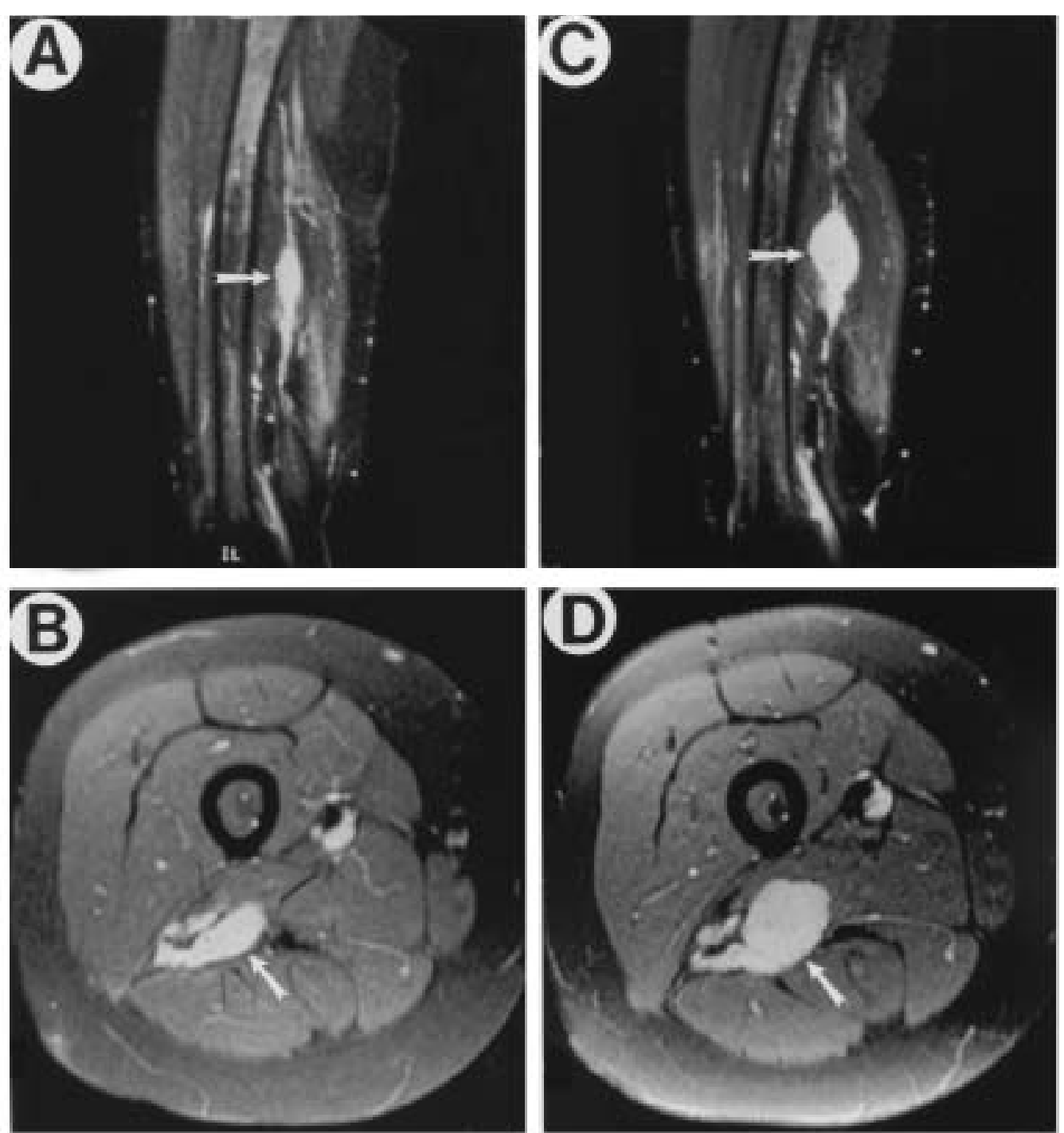

FIG. 10. Gadolinium-enhanced T1-weighted MR images obtained in a 9-year-old boy with a symptomatic mass (white arrows) of the right sciatic nerve at thigh level. The initial diagnosis was benign schwannoma. Sagittal (A) and axial (B) MR images showing the sciatic nerve after a partial resection of the mass. Follow-up sagittal (C) and axial (D) MR images obtained 1.5 years later showing enlargement of the residual mass (which was later resected) and the sciatic nerve repaired with multiple nerve grafts. The final pathological diagnosis was consistent with an intermediate grade of sciatic nerve sheath tumor.

we do not advocate such an approach, favoring instead open biopsy and resection of a tumor to avoid damaging functioning nerve fibers (which can cause significant pain, as well as weakness and numbness).

Postoperatively, imaging is used to determine the extent of resection performed to verify if it was total or partial. Subsequent follow-up imaging helps in identifying growth of residual tumor, as well as determining if there is tumor recurrence, particularly if there are new clinical symptoms and findings (Fig. 10).

\section{CONCLUSIONS}

Although imaging alone might not be sufficient to provide an accurate diagnosis of a peripheral nerve mass, it plays a critical role. Among all imaging methods, MR imaging is the preferred modality for evaluating peripheral nerve tumors and other lesion types. In some cases, the appearance of a mass can be unique enough to provide a strong presumptive diagnosis. However, imaging cannot reliably determine the pathological type of a tumor and thus cannot differentiate between benign and malignant masses or distinguish one type of tumor from another. Nevertheless, imaging can provide a very useful roadmap to the physician evaluating peripheral nerve lesions and can assist in navigating a safe and effective clinical course.

\section{References}

1. Beekman R, Visser LH: High-resolution sonography of the peripheral nervous system-a review of the literature. Eur J Neurol 11:305-314, 2004 
2. Bhargava R, Parham DM, Lasater OE, Chari RS, Chen G, Fletcher BD: MR imaging differentiation of benign and malignant peripheral nerve sheath tumors: use of the target sign. Pediatr Radiol 27:124-129, 1997

3. Britz GW, Goodkin R, Kliot M: Peripheral nerve tumors: pathologic, diagnostic, and treatment considerations, in Grossman RG, Loftus CM (eds): Principles of Neurosurgery. Philadelphia: Lippincott-Raven Press, 1999, pp 665-678

4. Britz GW, Lee JC, Goodkin R, Kliot M: Peripheral nerve tumors, in Keating RF, Goodrich JT, Packer RJ (eds): Tumors of the Pediatric Central Nervous System. New York: Stuttgart Thieme, 2001, pp 453-463

5. Cerofolini E, Landi A, DeSantis G, Maiorana A, Canossi G, Romagnoli R: MR of benign peripheral nerve sheath tumors. J Comput Assist Tomgr 15:593-597, 1991

6. Das Gupta TK, Chaudhuri PK: Tumors of the peripheral nerves, in Das Gupta TK, Chaudhuri PK (eds): Tumors of the Soft Tissues. New York: McGraw-Hill, 1998, pp 343-396

7. Donner TR, Voorhies RM, Kline DG: Neural sheath tumors of major nerves. J Neurosurg 81:362-373, 1994

8. Filler AG, Howe FA, Hayes CE, Kliot M, Winn HR, Bell BA, et al: Magnetic resonance neurography. Lancet 341:659-661, 1993

9. Grant GA, Britz GW, Goodkin R, Jarvik JG, Maravilla K, Kliot M: The utility of magnetic resonance imaging in evaluating peripheral nerve disorders. Muscle Nerve 25:314-331, 2002

10. Grant GA, Goodkin R, Kliot M: Evaluation and surgical management of peripheral nerve problems. Neurosurgery 44: 825-840, 1999

11. Guha A, Bilbao J, Kline DG, Hudson AR: Tumors of the peripheral nervous system, in Youmans JR (ed): Neurological Surgery: A Comprehensive Reference Guide to the Diagnosis and Management of Neurosurgical Problems. Philadelphia: WB Saunders, 1996, pp 3175-3187

12. Hain SF, O'Doherty MJ, Bingham J, Chinyama C, Smith MA: Can FDG PET be used to successfully direct preoperative biopsy of soft tissue tumors? Nucl Med Commun 24:1139-1143, 2003

13. Kline DG, Donner T, Voorhies R: Management of tumors involving peripheral nerves, in Tindall GT, Cooper PR, Barrow DK (eds): The Practice of Neurosurgery. Baltimore: Williams \& Wilkins, 1996, pp 1359-1369

14. Kransdorf MJ, Murphey MD: Imaging of soft tissue tumors, in Das Gupta TK, Chaudhury PK (eds): Tumors of the Soft Tissues, ed 2. Stamford: Appleton \& Lange, 1998, pp 38-79

15. Kuntz C IV, Blake L, Britz G, Filler A, Hayes CE, Goodkin R, et al: Magnetic resonance neurography of peripheral nerve lesions in the lower extremity. Neurosurgery 39:750-757, 1996
16. Kwok K, Slimp J, Born D, Goodkin R, Kliot M: The evaluation and management of benign peripheral nerve tumors and masses, in Berger MS, Prados MD (eds): Textbook of Neuro-Oncology. Philadelphia: Elsevier Saunders, 2005, pp 535-563

17. Leijten FS, Arts WF, Puylaert JB: Ultrasound diagnosis of an intraneural ganglion cyst of the peroneal nerve. Case report. J Neurosurg 76:538-540, 1992

18. Levine E, Huntrakoon M, Wetzel LH: Malignant nerve-sheath neoplasms in neurofibromatosis: distinction from benign tumors by using imaging techniques. AJR Am J Roentgenol 149:1059-1064, 1987

19. Lin J, Martel W: Cross-sectional imaging of peripheral nerve sheath tumors: characteristic signs on CT, MR imaging, and sonography. AJR Am J Roentgenol 176:75-82, 2001

20. Lusk MD, Kline DG, Garcia CA: Tumors of the brachial plexus. Neurosurgery 21:439-453, 1987

21. Mafee MF, Coombs RJ, Shinaver CN, Woldenburg LS: Imaging of soft tissue sarcomas, in Das Gupta TK, Chaudhuri PK (eds): Tumors of the Soft Tissues, ed 2. Stamford: Appleton \& Lange, 1998, pp 37-62

22. Maravilla KR, Bowen BC: Imaging of the peripheral nervous system: evaluation of peripheral neuropathy and plexopathy. AJNR Am J Neuroradiol 19:1011-1023, 1998

23. Martinoli C, Bianchi S, Derchi LE: Ultrasonography of peripheral nerves. Semin Ultrasound CT MR 21:205-213, 2000

24. Powers SK, Norman D, Edwards MS: Computerized tomography of peripheral nerve lesions. J Neurosurg 59:131-136, 1983

25. Senchenkov A, Kriegel A, Staren ED, Allison DC: Use of intraoperative ultrasound in excision of multiple schwannomas of the thigh. J Clin Ultrasound 33:360-363, 2005

26. Stull MA, Moser RP Jr, Kransdorf MJ, Bogumill GP, Nelson $\mathrm{MC}$ : Magnetic resonance appearance of peripheral nerve sheath tumors. Skeletal Radiol 20:9-14, 1991

27. Suh JS, Abenoza P, Galloway HR, Everson LI, Griffiths HJ: Peripheral (extracranial) nerve tumors: correlation of MR imaging and histologic findings. Radiology 183:341-346, 1992

28. Tindall SC: Ganglion cysts of peripheral nerves, in Wilkins RH, Rengachary SS (eds): Neurosurgery, ed 2. New York: McGraw-Hill, 1996, pp 3225

Manuscript received March 23, 2007.

Accepted in final form April 10, 2007.

Sources of support: Merit Review VA Grant.

Address reprint requests to: Michel Kliot, M.D., University of Washington Medical Center Department of Neurological Surgery, 1959 NE Pacific, Box 356470, Seattle, Washington 98195. email: Kliot@u.washington.edu. 